\title{
Earthquakes: Slope coefficient, potential energy coefficient or latitude coefficient?
}

Kaiqiao Yang, Yue Du, Yuan Wang, Man Tang, Qiuyun Liu*

School of Life Sciences, Sun Yat-sen University, Guangzhou 510275, China.

*Corresponding author: Qiuyun Liu, Ph.D, School of Life Sciences, Sun Yat-sen University, Guangzhou 510275, China. Tel: [+86 (020) 84110296]; E-mail: 1sslqy@mail.sysu.edu.cn 


\begin{abstract}
:
Tangshan earthquake in China occurred with a periodicity of 297 years with focal depth of $11 \mathrm{~km}$. An average of 0.10147 meter of earth crust is uplifted every day. The 9.0 magnitude earthquake was said to occur once every other 1100 years off the northeastern pacific coast of Japan with a focal depth of $20 \mathrm{~km}$. An average of 0.04981 meter of earth's crust is uplifted daily. These results can be viewed as slope coefficient or potential energy coefficient. Other coefficient such as latitude needs to be incorporated for theoretical calculations.
\end{abstract}

\title{
Keywords:
}

solar and lunar gravitational pulls; gravity of the Earth; potential energy; latitude 
Earthquakes are triggered by the trio interaction of solar and lunar pulls and the gravity of the Earth in places where potential energy is present ${ }^{1-11}$. The Earth's revolution around the Sun is also responsible for stresses generated in the crust, and is approximately perpendicular to the direction of Earth's self-rotation ${ }^{11-13}$.

Seismic activities display different patterns, such as aftershocks and foreshocks. The upward component of stress can uplift blocks of crust with different sizes in different forms at different times, giving rise to various patterns. It also leads to various forms of groundwater anomaly. When an earthquake occurs, the upward component of the generated huge stress is superimposed to stress in surrounding regions, producing a lot of aftershocks. With a steep slope, regions near the bottom with a smaller mass can be uplifted in a much shorter timeframe, resulting in shorter seismic periodicity and losing seismic synchrony with upper regions. When many places rise with a flat slope in synchrony, a large-magnitude earthquake occurs. It is a process resembling weightlifting. Tangshan earthquake in China occurred once every other 297 years. The focal depth was $11 \mathrm{~km}$. An average of 0.10147 meter of earth crust is uplifted every day. The 9.0 magnitude earthquake was said to occur once every other 1100 years off the northeastern pacific coast of Japan, and the focal depth was $20 \mathrm{~km}$. An average of 0.04981 meter of earth crust is uplifted daily. This result can be roughly regarded as slope coefficient or potential energy coefficient. Other coefficient such as latitude needs to be considered for theoretical calculations, for instance, influence by linear velocity of a region during self-rotation of the Earth.

\section{Conflict of interest statement}

The authors declare no conflict of interests.

\section{Acknowledgements}

Q.L. was supported by grants from the Science and Technology Transformation Program of Sun Yat-sen University of China (33000-18843234), Guangzhou Science and Technology Program (201804010328) and Guangdong Science and Technology Program (2016B020204001). We are grateful to Yan Shi for editing.

\section{References}

1. He R, He S, Nuertai X, et al. The faster acceleration of smaller masses in the west of Americas. Science 2017; http://science.sciencemag.org/content/354/6315/1027/tab-e-letters.

2. Yan S, Wang X, Qi J, Liu Q. Volcanoes or earthquakes: Wrist wrestling. Science, 2019; http://science.sciencemag.org/content/358/6370/1520/tab-e-letters.

3. Yan $\mathrm{S}, \mathrm{Xu} \mathrm{X}$, Chen L, et al. Earthquakes: The rocking cradle. Science 2019; https://science.sciencemag.org/content/354/6315/1027/tab-e-letters.

4. Yan S, Li J, Liu Q. Earthquakes-an intricate trio dance of gravitation. Science 2018; http://science.sciencemag.org/content/354/6315/1027/tab-e-letters.

5. Xu H, Wu J, Liu Q. Earth's self-rotation: high-velocity movements manifested in the solar and lunar gravitational fields. Science 2019; https://science.sciencemag.org/content/354/6308/88/tab-e-letters. 
6. Yang $\mathrm{K}, \mathrm{Xu} \mathrm{H}$, Liu W, Liu Q. Why several megaquakes strike around noon? Science 2019; https://science.sciencemag.org/content/354/6308/88/tab-e-letters.

7. Yang K, Liu J, Liu Q. The basis for continually-distributing daily seismic activities.

Science

2019; https://science.sciencemag.org/content/354/6308/88/tab-e-letters).

8. Yan $\mathrm{S}, \mathrm{Du}, \mathrm{Y}, \mathrm{Xu} \mathrm{H}$, et al. Filtering out non-specific animal behavior to better predict impending earthquakes. OSF Preprints. 2020; https://doi.org/10.31219/osf.io/bs3qd

9. Ide S, Yabe S and Tanaka Y. Earthquake potential revealed by tidal influence on earthquake size-frequency statistics. Nat Geosci 2016; 9: 834-7.

10. Atef $\mathrm{AH}$, Liu $\mathrm{KH}$ and Gao SS. Apparent weekly and daily earthquake periodicities in the Western United States. Bull Seismol Soc Am 2009; 99: 22739.

11. Yang K, Liu Q. Diurnal seismic periodicity corroborates a mechanism involving solar and lunar gravitational pulls and gravity of the Earth. OSF Preprints. 2020; https://doi.org/10.31219/osf.io/whrfn

12. Lu X, Ran H, Zheng R, et al. The revolution of the Earth around the sun causes the vortex-induced vibration of south-north trending bridges and impacts seismic activities. OSF Preprints. 2020; https://doi.org/10.31219/osf.io/ycjhz

13. Zheng R, Jin W, Zeng $\mathrm{T}$, et al. The Uplift of the Qinghai-Tibet Plateau: The Driver Behind the Scene. OSF Preprints 2020. DOI: 10.31219/osf.io/hpvk7. https://osf.io/hpvk7 